\title{
Colloid versus crystalloids in shock
}

\section{Ken Hillman}

This is a review of the use of colloid and crystalloid in shock. The article discusses the pathophysiological
consequences of ischaemia and shock; reviews the underlying physiology of fluid compartments and how
they may be affected by disease; examines the physiological determinants of intravenous (IV) fluid use; and
finally reviews the literature on colloid and crystalloid use for the management of shock.

Key Words: Shock, Fluid resuscitation, Colloids, Crystalloids
The debate about whether to use crystalloids or colloids for the resuscitation of hypovolaemia is not as important as the challenge of continually maintaining a normal intravascular volume. In this article we will discuss hypovolaemia and its consequences, the physiology of body fluids and finally the choice of the most appropriate fluid, touching on the colloid-crystalloid controversy.

Hypovolaemia is one of the most common and potentially reversible crises in acute medicine. It occurs as the result of fluid loss (e.g. bleeding, burns, vomiting and diarrhoea) or vasodilatation of the circulating volume (e.g. septic shock). In either case, rapid correction is mandatory. In the setting of Intensive Care, there is the initial challenge of shock and resuscitation but there is also the often overlooked challenge of maintaining euvolaemia over the ensuring days.

In the daily routine of Intensive Care we continually monitor hypovolaemia with vital signs such as blood pressure (BP) and pulse rate (PR) as well as by monitoring end organ function such as urine output and peripheral perfusion. Even minor degrees of hypovolaemia can cause ischaemia and organ dysfunction. ${ }^{1}$ It is therefore, not surprising, that we are constantly on the alert for the

\section{From:}

Professor of Intensive Care, University of New South Wales, Sydney, Australia. Correspondence:

Prof. Ken Hillman

Division of Critical Care, Liverpool Hospital, Locked Bag - 7103, Liverpool BC, NSW - 1871, Australia. E-mail: K.Hillman@unsw.edu.au first signs of hypovolaemia, and then having detected it, rapidly correcting any deficit. The usual strategy in the Intensive Care Unit (ICU) is to either increase the rate of IV fluid infusion or deliver a bolus of $300-400 \mathrm{ml}-\mathrm{a}$ fluid challenge - assess the effect and adjust the fluid rate accordingly.

The importance of maintaining the intravascular space (IVS) is related to the deleterious effects of ischaemia. There is no conclusive data on exactly how much ischaemia can be safely tolerated. Particularly important are cells with high metabolic rates such as the brain, heart and kidney. These organs initially have their blood supply protected by autoregulation at the expense of decreased blood supply to non-vital organs such as the skin and muscle. Ischaemia can be better tolerated in organs such as the skin or muscle as they have a low metabolic rate. However, there comes a point when even these cells begin to malfunction. Even minor degrees of hypovolaemia predisposes to splanchnic hypoperfusion, ${ }^{2}$ predisposing to translocation of bacteria and bacterial breakdown products. ${ }^{3}$ This can in turn predispose to multiorgan dysfunction syndrome (MODS) and even death..$^{3-6}$ Cytokines are also released as a result of the ischaemia itself, adding to the MODS. ${ }^{7,8}$

The concept of early detection and correction of ischaemia, as well as rapid establishment of an airway and delivery of adequate oxygen delivery is the basis of the 'Golden Hour', emphasising the importance of rapid resuscitation in order to prevent cellular damage. The 
so-called 'Golden Hour' applies not only to initial resuscitation but also to continually maintaining the IVS, while the patient is in the ICU.

There is no proven place for deliberately tolerating even the smallest degree of ischaemia. While deliberate hypoventilation or permissive hypercarbia is a proven strategy to protect overventilation of lungs and damage to alveoli; permissive hypercarbia does not mean permissive hypoxia. And permissive hypercarbia and its benefits do not equate to permissive hypotension, which in fact is "permissive shock and ischaemia". In special circumstances such as penetrating injury to the torso, it may be better to perform definitive surgery rapidly rather than attempt to restore circulating volume..$^{9}$ This does not mean that ischaemia is an acceptable state. It simply means that early definitive surgery is probably a better option in that relatively unique clinical circumstance. Unfortunately, because surgery may be a higher priority than IV resuscitation in this particular and relatively rare circumstance, there has been an extropolation to other situations, inferring that ischaemia is acceptable in other circumstances. This is not the case.

\section{The Body's Fluid Spaces}

There are approximately $40 \mathrm{I}$ of water in a $70 \mathrm{~kg}$ adult male $-60 \%$ of the body's weight. ${ }^{10}$

In the newborn it is closer to $80 \%$ and in the very old, approximately $40 \%$. In other words, the young require higher maintenance fluid and as you age, you contain less water.

The water is distributed between three spaces, each with a distinct function and set of physiological principles governing its volume - the IVS; the interstitial space (ISS); and the intracellular space (ICS).

\section{Intravascular Space}

The IVS contains about 5 I of blood - red cells and plasma. The circulation delivers nutrients and oxygen to cells and removes carbon dioxide and products of metabolism. The IVS is contained within the circulation by the endothelial cells which, while allowing some leakage of large molecules such as proteins, largely encourages their retention within the IVS, generating a colloid oncotic pressure (COP), which encourages fluid movement from the interstitial space (ISS), counteracting the effect of the hydrostatic pressure within the capillaries.

\section{Interstitial Space}

The ISS contains about 12 I of water and represents the fluid between the IVS and cells, facilitating transport between the two and, because of its connective tissue, contributing to the general structure and shape of the body. Apart from water, the ISS contains electrolytes, with the predominate cation being sodium $(\mathrm{Na})$ in the same concentration as the IVS. Sometimes the ISS and IVS are together labelled as the extracellular fluid (ECF). This is an artificial construct as the IVS and ISS have very different functions and constituents.

\section{Intracellular Space}

The ICS is the largest space (approximately $23 \mathrm{I}$ ) and has potassium $\left(\mathrm{K}^{+}\right)$as its major cation. Therefore, sodium containing fluids will therefore not be distributed to the ICS.

\section{Assessing the Fluid Space Intravascular Space}

The IVS is the most amenable to measurement. There are two major ways we can achieve that. Firstly, the global capacity of the IVS is estimated by measurements such as systemic BP; PR; central venous pressure (CVP) and jugular venous pressure (JVP); pulmonary artery wedge pressure (PAWP); Pulse Contour Cardiac Output (PCCO); and cardiac output (CO).

All of these measurements have limitations. The major one is that they are estimations of global blood flow and not the more important measures of organ perfusion. Bearing this shortcoming in mind, global measurements nevertheless give an indication of the adequacy of the circulating volume when looked at together with measures of organ perfusion and in the light of the clinical setting.

Systemic BP tracks the IVS more accurately in the elderly as they tend to have less of a sympathetico-adrenal response which allows younger patients to maintain $\mathrm{BP}$ until almost total circulatory collapse has occurred.

Similarly with PR - younger patients will mount a more vigorous tachycardia in response to hypovolaemia. The CVP may increase with heart failure or decrease with hypovolaemia. However, younger patients generate a 
more active venoconstriction as a result of hypovolaemia, resulting in an increase in CVP or JVP, compared to the elderly. There are similar shortcomings related to pathophysiological assumptions with the use of PAWP and $\mathrm{CO}$, resulting in widespread reduction in the use of pulmonary artery catheters in the ICU.

The PCCO System has been developed, where continuous estimation of cardiac output is made by using an arterial pulse contour algorithm via a peripheral arterial line and trace. ${ }^{11}$ The system is initially calibrated using a transpulmonary thermodilution measurement. A bolus of intravascular fluid is injected through a central venous catheter and the curve recorded by an arterial thermodilution catheter, which also serves as a pressure monitoring source. ${ }^{12}$ The system also estimates intrathoracic blood volume; global end-diastolic volume; extravascular lung water; pulmonary vascular permeability index; and global ejection fraction. ${ }^{13-15}$

Another way of estimating adequacy of circulatory volume is by determining peripheral organ perfusion. Vital organs such as the heart and brain have their blood supply preserved even at very low levels of IVS and so are late indicators of indicators of the IVS, often immediately preceding death, especially in the young.

The kidney, also a vital organ, is, however, a very useful organ to determine adequacy of the circulating volume. Hourly urine output measurement is one of the simplest and most accurate ways of determining adequacy of the IVS. Moreover, renal failure as a result of hypovolaemia has such disastrous and expensive consequences that it should be avoided, where possible, by the simple administration of IV fluid. Obviously renal perfusion and urine output can also be affected by cardiac failure and, as such, needs to be interpreted in the light of other clinical findings. However, even with a failing heart, decreased urine output may result from inadequate volume as the filling pressure of the heart in these circumstances needs to be high. Decreased urine output can also be caused by other factors in the setting of an ICU, such as underlying renal disease or drugs such as diuretics.

Another organ which can reflect hypovolaemia is the skin. Hypovolaemia is detected by pressure receptors in the circulation resulting in increased sympathetico-ad- renal activity, which in turn results in vasoconstriction of many of the less vital organs, including the skin. Early hypovolaemia is reflected in cooling of the most peripheral parts of the limbs - i.e. fingers and toes. Obviously pump failure can have the same effect as hypovolaemia and so the clinical setting needs to be taken into account.

The circulation is a dynamic organ controlled by many complex interactions. Rather than estimate the IVS with one measurement at one point in time, it is better to use as many of the measurements as possible and consider them as trends and to dynamically determine the adequacy of filling by a fluid challenge - i.e. administering 200-300 ml fluid rapidly, and measuring the response of the observations both before and after the challenge. ${ }^{16}$ If the measurements improve, then repeat the challenge and continue the fluid challenges until improvement in measurements is minimal. This point is an indication of an ideal IVS. Obviously the state of the circulation will be continually changing, necessitating close observation, repeated challenges and appropriate action.

Maintaining an appropriate IVS at all times is so important that perhaps the clinician who continually cares for the patient (usually a nurse) should be instructed on how to deliver fluid challenges and adequate fluid volumes as soon as any signs of hypovolaemia are noted. Obviously they would need to refer to their medical colleagues when there is any doubt.

\section{Interstitial and Intracellular Space}

Although these spaces are much larger than the IVS, they are less amenable to measurement. 'Softer' and less accurate measures such as thirst and mucosal dryness have to be used. The most accurate indicator of the ISS is probably peripheral oedema. This is easily assessed at the bedside. In clinical practise, the most accurate indicator of total body water is the serum sodium. ${ }^{17}$ The total body water is, in turn, the best guide to the ICS as the majority of body fluid is contained within cells. The reasons for serum sodium being an accurate indicator of body water is related to the balance between water and sodium in determining the concentration levels of sodium. The serum sodium concentration is, almost always, determined by total levels of body water, rather than the total levels of body sodium. 


\section{History of Fluid Administration}

The first IV fluid was administered in 1832 for a cholera victim. ${ }^{18}$ Shock was a term first used to describe war casualties to describe their appearance as they seemed to die as a result of hypovolaemia, rather than as a direct result of their injuries. Cournand ${ }^{19}$ first described physiological abnormalities of shock. Shire ${ }^{20}$ advocated the use of large amounts of crystalloids in the postoperative period in the 1950s. This was probably a reasonable approach as renal failure as a result of hypovolaemia was at that time, invariably fatal. As a result most postoperative patients were overtransfused with large amounts of crystalloids, usually charted in an inflexible way, in order to avoid renal failure in the small number of patients who had abnormal perioperative fluid losses.

Today's postoperative fluid prescribing is a legacy of that period - large amounts of crystalloid are administered in the postoperative period in a wide variety of inflexible recipes with little regard to the nature of fluid needs of the patient. Fortunately most operations are without complication and the body's finely tuned mechanisms for responding to fluid overload usually prevents serious complications.

The use of crystalloids in the postoperative period was then taken up for resuscitation of the seriously ill in ICUs. ${ }^{1}$ Moreover, there were very few colloid solutions available at that time. Another factor for the failure to seriously question the approach to fluid therapy first developed in the 1960 's is that with increasing specialisation in medicine, fluid and electrolyte therapy has not had the critical attention applied to its scientific foundations as, for example, if the field was confined to a single free standing organ such as the heart, gastrointestinal tract, lungs, brain, etc. In other words, important several body functions such as fluids and electrolytes and the lymphatics are not owned by any single specialty and therefore have tended to be neglected in terms of research and a critical appraisal of their role in acute medicine.

\section{Crystalloids or Colloids}

Colloids are mainly distributed to the IVS. ${ }^{10}$ Even with increased capillary leakage, a greater proportion of a colloid solution will remain in the IVS compared to a crystalloid solution.

On the other hand crystalloid solutions are mainly dis- tributed to the ISS. Approximately two-thirds of all infused crystalloid must be distributed to the ISS, according to basic physiology, ie there are $17 \mathrm{I}$ of fluid in the ECF and a crystalloid solution, without any inherent COP, must distribute equally across the whole 17 litres, with little or no fluid going to the ICS. ${ }^{21}$

In other words if the IVS is underfilled according to all the measurements we have, then a fluid that is distributed to that space such as blood or colloid is the ideal fluid. To use a crystalloid (which is mainly distributed to the ISS), according to measurements of a different space (the IVS), does not make physiological sense.

Moreover, the ISS is rarely underfilled in the seriously ill. In fact, most patients in the ICU have peripheral oedema. Giving crystalloids in these circumstances would add to peripheral oedema.

\section{Disadvantages of an Expanded Interstitial Space and Crystalloid use}

As little as 1 litre of normal or isotonic saline adversely affects pulmonary function by increasing closing volume and decreasing static and dynamic compliance. ${ }^{22}$ Increasing volumes of crystalloid result in overt hypoxia and increasing degrees of pulmonary oedema. ${ }^{23-26}$ This is not due to heart failure but simply due to the natural compartmentalising of saline into the ISS.

As well as expansion of the ISS in the lungs, peripheral oedema also occurs. ${ }^{24,25,27}$ Peripheral oedema can markedly decrease oxygen consumption. ${ }^{28}$ This may be related to increased distance between the cells and capillary or be as a result of occlusion of the thin walled capillaries by an increase in the surrounding ISS pressure. This may explain why colloids have been demonstrated to improve tissue oxygenation compared to crystalloids. ${ }^{29}$

Peripheral oedema also results in delayed wound healing. ${ }^{30}$ Doubling the volume of the ISS decreases oxygen delivery to such an extent that capillary blood flow needs to be increased by 20 -fold in order to maintain the same oxygen tension. ${ }^{31}$

Crystalloids result in a hypercoagulable state which can result in increased incidence of intravascular thrombosis. 32,33 
A significant hyperchloraemic acidosis can occur when large amounts of isotonic saline are used..$^{34}$ The use of Ringer's Lactate is not associated with this phenomenon. ${ }^{35}$

\section{Clinical differences between Colloids and Crystalloids}

There is compelling physiological evidence to use colloids for resuscitation of hypovolaemia. But what of clinical evidence?

In most cases the clinical differences between the two fluids may be marginal. The patients own adaptive neuroendocrine systems senses fluid overload and, with intact kidneys, will correct it. Moreover the lymphatic system also becomes a hero of fluid abuse. They drain excessive fluid from the ISS, thus limiting fluid expansion which would otherwise result in peripheral and pulmonary oedema. ${ }^{36}$

As expected, clinical differences in the amount of peripheral oedema are accentuated with crystalloids compared to colloids. ${ }^{37-40}$

Similarly, with pulmonary oedema, crystalloids cause greater oedema and hypoxia. ${ }^{24,41-43}$ There is also a good evidence that increased leaky capillaries in the lungs does not occur with sepsis ${ }^{24,26}$ and that it is more likely related to simple overexpansion of the ISS by excessive crystalloid solution.

However, using extreme end-points such as mortality, some larger comparative studies favour crystalloids ${ }^{44-47}$ and other studies favour colloids. ${ }^{26,48-51} \mathrm{~A}$ meta-analysis of colloid versus crystalloid studies in $1989^{52}$ demonstrated that pooled data from eight studies showed no difference. However, in the eight studies which did not use mortality as an end-point, six showed colloids to be more efficacious, one showed more benefit from crystalloids and one showed no difference.

More recent meta-analysis studies have been similarly inconclusive. Some have clearly favoured crystalloid therapy. ${ }^{53}$ In a Cochrane Review, there was no evidence that resuscitation with colloids reduced the risk of death in patients with trauma, burns and following surgery. ${ }^{54}$ When albumin was used as the colloid solutions, there was no overall benefit of that particular colloid. ${ }^{55}$ The use of meta-analysis can be questioned as most studies do not use mortality as the end-point. ${ }^{56}$

It seems obvious that we need more original research in this area rather than attempting to match all trials with new statistics ${ }^{57}+52-$ see $B$ attached.

\section{Conclusion}

No matter what the cause of hypovolaemia, e.g. trauma, sepsis, excessive GIT losses, diabetic ketoacidosis, the first priority is to rapidly restore the circulation.

There are many patients in acute hospitals who remain hypovolaemic either in general wards or in Emergency Departments for long periods before they are transferred to ICUs. ${ }^{59-63}$

For these patients, expensive drugs or magic bullets aimed at counteracting the deleterious effects of cytokines after they are released, have little or no effect. ${ }^{64-67}$

Early detection and rapid reversal of ischaemia and hypoxia is a cheaper and more logical approach. ${ }^{68}$ To this end a hospital-wide approach has been developed, where abnormalities in vital signs such as hypotension and tachycardia cause activation of a Medical Emergency Team (MET). ${ }^{69-73}$ The MET is organised by the ICU and replaces the hospital's cardiac arrest team. It is called to easily measured signs measured at most patient's bedside (e.g. system BP $<90 \mathrm{mmHg}$; $P R<40>140$ rate/min; respiratory rate $<4>36$ rate/min; or sudden loss of consciousness). The aim is early resuscitation and restoration of a normal circulatory volume rather than admission to the ICU at a later stage when the patient may have MODS with a poorer outcome.

For high risk patients, the rapidity of replacement of fluid is more important than the actual type of fluid administered. The colloid/crystalloid controversy is largely an aritificial construct. If the ISS is dry use a crystalloid and if the IVS is dry, use a colloid.

\section{References}

1. Hillman K, Bishop G, Bristow P. The crystalloid versus colloid controversy: Present status. In: Halkjamae H, editor. Baillier's Clinical Anaesthesiology. International Practice and Research. Plasma Volume Support. London: Bailliere Tindall; 1997;11:1- 


\section{ISBN 0-7020-2341-8.}

2. Price HL, Deutsch S, Marshal BNE, et al. Hemodynamic and metabolic effects of hemorrhage in man, with particular reference to the splanchnic circulation. Circulation Research 1966;18:469-74.

3. Koziol JM, Rush BJ Jr, Smith SM, Machiedo GW. Occurrence of bacteremia during and after hemorrhagic shock: Efficacy and cardiopulmonary consequences. Crit Care Med 1988;28:10-6.

4. Biffl WI, Moore EE. Splanchnic ischaemia/reperfusion and multiple organ failure. Br J Anaesth 1996;77:59-70.

5. Mythen MG, Webb AR. Intra-operative gut mucosal hypoperfusion is associated with increased post-operative complications and cost. Intensive Care Med 1994;20:99-104.

6. Takala J. Splanchnic perfusion in shock. Intensive Care med 1994;20:403-4.

7. Rush BJ. The bacterial factor in hemorrhagic shock. Surg Gynae Obstet 1992;175:285-92.

8. Bernard GR. Research in sepsis and acute respiratory distress syndrome: Are we changing course? Crit Care Med 1999;27:434-6.

9. Bicknell WH, Wall MJ, Pepe PE, Martin RR, Ginger VF, Allen $\mathrm{MK}$, et al. Immediate versus delayed fluid resuscitation for hypotensive patients with penetrating torso injuries. $\mathrm{N}$ Engl $\mathrm{J}$ Med 1994;331:1105-9.

10. Hillman K. Fluids and electrolytes. In: Scurr C, Felman S, Soni N, editors. Scientific Foundations of Anaesthesia, $4^{\text {th }}$ Ed. Heinemann: Medical Books; 1990. p. 448-62.

11. Böck JC, Barker BC, Mackersie RC, Tranbaugh RF, Lewis FR. Cardiac output measurement using femoral artery thermodilution in patients. J Crit Care 1989;4:106-11.

12. Sakka SG, Reinhart K, Meier-Helimann A. Comparison of pulmonary arterial and arterial thermodilution cardiac output in critically ill patients. Intensive Care Med 1999;25:843-6.

13. Lichtwarck-Aschoff M, Zeravik J, Pfeiffer UJ. Intrathoracic blood volume accurately reflects circulatory volume status in critically ill patients with mechanical ventilation. Intensive Care Med 1992;18:137-8.

14. Hedenstierna $G$. What value does the recording of intrathoracic blood volume have in clinical practice? Intensive Care Med 1992;18:137-8.

15. Laggner A, Kleinberger G, Hailer J, Lenz K, Sommer G, Drumi $W$. Bedside estimation of extravascular lung water in critically ill patients: Comparison of the chest radiograph and the thermal dye technique. Intensive Care Med 1984;10:309-13.

16. Weil MH, Henning RJ. New concepts in the diagnosis and fluid treatment of circulatory shock. Anesth Analg 1979;58:124-32.

17. Parkin G. Circulatory disorders: Intervention and control. Measurement in Medicine 1986;1:313.

18. Cosnett JE. The origins of intravenous fluid therapy. Lancet 1989;i:768-71.

19. Cournard A, Riley RL, Bradley SE, et al. Studies of the circulation in clinical shock. Surgery 1943;13:964-95.

20. Shires GT, Williams J, Brown F. Acute changes in extracellular fluids associated with major surgical procedures. Ann Surg 1961;154:803-10.

21. Twigley AJ, Hillman KM. The end of the crystalloid era? A new approach to peri-operative fluid administration. Anaesthesia 1985;40:860-71.

22. Collins JV, Cochran GM, Davis J. Some aspects of pulmonary function after rapid saline infusion in health subjects. Clinical Science 1973;45:4907-10.

23. Shoemaker WC. Comparison of the relative effectiveness of whole blood transfusions and various types of fluid therapy in resuscitation. Crit Care Med 1976;4:71-8.

24. Hauser CJ, Shoemaker WC, Turpin I, Goldberg SJ. Oxygen transport responses to colloids and crystalloids in critically ill surgical patients. Surg Gyne Obstet 1980;150:811-6.

25. Brinkmeyer S, Safar P, Motoyama E, Stezoski W. Superiority of colloid over electrolyte solutions for fluid resuscitation (sever normovolemic hemodilution). Crit Care Med 1981;9:369-70.

26. Rackow EC, Falk JL, Fein IA, et al. Fluid resuscitation in circulatory shock: A comparison of the cardiorespiratory effects of albumin, hetastarch, and saline solutions in patients with hypovolemic and septic shock. Crit Care Med 1983;11:839-50.

27. Baun TD, Wang H, Rothschild HR, et al. Mesenteric oxygen metabolism, ileal mucosal hydrogen ion concentration, and tissue edema after crystalloid or colloid resuscitation in porcine endotoxic shock: Comparison of Ringer's lactate and 6\% hetastarch. Circulatory Shock 1990;30:385-97.

28. Dawidson I, Eriksson B. Statistical evaluation of plasma substitutes based on 10 variables. Crit Care Med 1982;10:653-7.

29. Lang K, Boldt J, Suttner S, Haisch G. Colloids versus crystalloids and tissue oxygen tension in patients undergoing major abdominal surgery. Anesth Analg 2001;90:1247-9.

30. Mangalore PP, Hunt TK. Effect of varying oxygen tensions on healing of open wounds. Surg Gyne Obstet 1972;135:756.

31. Knisely MH, Reneau DD, Binely DF. The development and use of equations for predicting limits on the rates of oxygen supply to the cells of tissues and organs. Angiology 1969;20:61.

32. Janvrin SB, Davies G, Greenhalph. Postoperative deep vein thrombosis caused by intravenous fluids during surgery. Br J Surg 1980;67:690-3.

33. Ng KFJ, Lam CCK, Chan LC. In vivo effect of haemodilution with saline on coagulation: A randomized controlled trial. $\mathrm{Br} \mathrm{J}$ Anaesth 2002;88:475-80.

34. Prough DS, Bidani A. Hyperchloremic metabolic acidosis is a predictable consequence of intraoperative infusion of $0.9 \%$ sa- 
line. Anesthesiology 1999;90:1247-9.

35. Takil A, Eti Z, Irmak P, Gögüs Y. Early postoperative respiratory acidosis after large intravascular volume infusion of lactated Ringer's solution during major surgery. Anesth Analg 2002;95:294-8.

36. Guyton AC, Taylor AE, Granger HJ, Coleman TG. Interstitial fluid pressure. Physiology Review 1971;51:527.

37. Schott U, Lindbom LO, Sjostrand U. Hemodynamic effects of colloid concentration in experimental hemorrhage. A comparison of Ringer's lactate, $3 \%$ dextran- 40 and $6 \%$ dextran-70. Crit Care Med 1988;16:346.

38. Rasmussen I, Arvidsson D, Zak A, Haglund U. Splanchnic and total body oxygen consumption in experimental fecal peritonitis in pigs: Effects of dextran and iloprost. Circulatory Shock 1992;36:299-306.

39. Dyess DL, Powell RW, Swafford AN, et al. Redistribution of organ blood flow after hemorrhage and resuscitation in full-term piglets. J Ped Surg 1994;29:1097-102.

40. Mythen MG, Webb AR. Perioperative plasma volume expansion reduces the incidence of gut mucosal hypoperfusion during cardiac surgery. Arch Surg 1995;130:423-9.

41. Stein L, Beraud JJ, Morissette M, et al. Pulmonary edema during volume infusion. Circulation 1975;52:483-9.

42. Finch JS, Reid C, Bandy K, Fickle D. Compared effects of selected colloids on extravascular lung water in dogs after oleic acid-induced lung injury and severe hemorrhage. Crit Care Med 1983;11:267-70

43. Shippy CR and Shoemaker WC. Hemodynamic and colloid osmotic pressure alternations in the surgical patient. Crit Care Med 1983;11:191-5.

44. Lucas GW, Waver D, Higgins RGF, et al. Effects of albumin versus non-albumin resuscitation on plasma volume and renal excretory function. J Trauma 1978;18:564-70.

45. Virgilio RW, Smith De, Zsarins CK. Balanced electrolye solutions: Experimental and clinical studies. Crit Care Med 1979;7:98-106.

46. Monafo WW. Volume replacement in hemorrhage, shock and burns. Advances in Shock Research 1980;3:47-56.

47. Poole GV, Meredith JW, Pennel T, Mills SA. Comparisons of colloids and crystalloids in resuscitation from hemorrhagic shock. Surg Gyne Obstet 1982;154:577-86.

48. Allardyce DB. Parenteral fluid therapy in septic shock: An evaluation of crystalloid and colloid. American Surgeon 1974;40: 542-7.

49. Dawidson I, Gelin I, Hedman L, Soderberg R. Hemodilution and recovery from experimental intestinal shock in rates: A comparison of the efficacy of three colloids and one electrolyte solution. Crit Care Med 1981;9:42-6.

50. Shoemaker WC, Schluchter M, Hopkins JA, et al. Comparison of the relative effectiveness of colloids and crystalloids in emer- gency resuscitation. Am J Surg 1981;9:367-68.

51. Modig J. Advantages of dextran 70 over Ringer acetate solution in shock treatment and in prevention of adult respiratory distress syndrome. A randomised study in man after traumatic hemorrhagic shock. Resuscitation 1983;10:219-26.

52. Velanovich V. Crystalloid versus colloid fluid resuscitation: A metaanalysis of mortality. Surgery 1989;105:65-71.

53. Schierhout G, Roberts I. Fluid resuscitation with colloids or crystalloids in critically ill patients: A systematic review of randomised trials. Br Med J 1998;316:961-4.

54. Cochrane Injuries Group Albumin Reviewers. Human albumin administration in critically ill patients - systematic review of randomised controlled trial. BMJ 1998;317:235-40.

55. Wilkes MM, Navickis RJ. Patient survival after human albumin administration - a meta-analysis of randomised controlled trials. Ann Intern Med 2001;135:149-64.

56. Astiz ME, Rackow EC. Crystalloid-colloid controversy revisited. Crit Care Med 1999;27:34-5.

57. Cook D, Guyatt G. Editorial: Colloid use for fluid resuscitation: evidence and spin. Ann Intern Med 2001;135:205-8.

58. Choi PT-L, Yip G, Quinonez LG, Cook DJ. Crystalloids vs colloids in fluid resuscitation: A systematic review. Crit Care Med 1999;27:200-10.

59. Gutierrez G, Palizas F, Doglio G, Wainsztein N, Gallesio A, Pacino J, et al. Gastric intramucosal pH as a therapeutic index of tissue oxygenation in critically ill patients. Lancet 1992;339:195-9

60. Hayes MA, Timmins AC, Yau EH, Palazzo M, Hinds CJ, Watson D. Elevation of systemic oxygen delivery in the treatment of critically ill patients. New Engl J Med 1994;330:1717-22.

61. Bishop MH, Shoemaker WC, Appel PL, Meade P, Ordog GJ, Wasserberger J, et al. Prospective, randomised trial of survivor values of cardiac index, oxygen delivery, and oxygen consumption as resuscitation endpoints in severe trauma. J Trauma 1995;38:780-7

62. Fleming A, Bishop M, Shoemaker W, Appel P, Sufficool W, Kurhenguwha $A$. Prospective trial of supranormal values as goals of resuscitation in severe trauma. Arch Surg 1992;127:1175-81.

63. Yu M, Levy MM, Smith P, Takiguchi SA, Miyasaki A, Myers SA. Effect of maximizing oxygen delivery on morbidity and mortality rates in critically ill patients: A prospective, randomised, controlled study. Crit Care Med 1993;21:830-8.

64. Bernard GR. Research in sepsis and acute respiratory distress syndrome: Are we changing course? Crit Care Med 1999;27:434-6.

65. Kirschenbaum L. Antibodies to TNF-á: Too little, too late? Crit Care Med 1998;26:1625-6.

66. Abraham E. Why immunomodulatory therapies have not worked 
in sepsis. Intensive Care Med 1999;25:556-66.

67. Delinger RP. Post hoc analyses in sepsis trials. A formula for disappointment? Crit Care Med 1996;24:727-9.

68. Hillman K, Parr M, Flabhouris A, Bishop G, Stewart A. Redefining in-hospital resuscitation: The concept of the medical emergency team. Resuscitation 2001;48:105-10.

69. Lee A, Bishop G, Hillman KM, Daffurn K. The medical emergency team. Anaesth Intensive Care 1995;23:183-6.

70. Hourihan F, Bishop G, Hillman KM, Daffurn K, Lee A. The medical emergency team: A new strategy to identify and intervene in high risk patients. Clin Intensive Care 1995;6:269-72.

71. Hillman KM, Bishop G, Lee A, Daffurn K, Bauman A, Crispin C, et al. Identifying the general ward patient at high risk of cardiac arrest. Clin Intensive Care 1996;7:242-3.

72. Hillman K. The changing role of acute-care hospitals. Med J Aust 1999;7:242-3

73. Hillman KM. Recognising and preventing serious in-hospital events. Med J Aust 1999;171:308-9.

\section{Abbreviations}

$\mathrm{BP}$

Blood pressure

CO Cardiac output

COP Colloid oncotic pressure

CVP Central venous pressure

ECF Extracellular fluid

ICU Intensive Care Unit

ICS Intracellular space

ISS Interstitial space

IV Intravascular

IVS Intravascular space

JVP Jugular venous pressure

$\mathrm{K}^{+} \quad$ Potassium

MODS Multiorgan dysfunction syndrome

$\mathrm{Na} \quad$ Sodium

PAWP Pulmonary artery wedge pressure

PCCO Pulse contour cardiac output

PR Pulse rate 